\title{
Chronic Achilles Tendon Rupture Reconstruction with a Endoscopic Flexor Hallucis Longus Transfer: A Case Re-Port
}

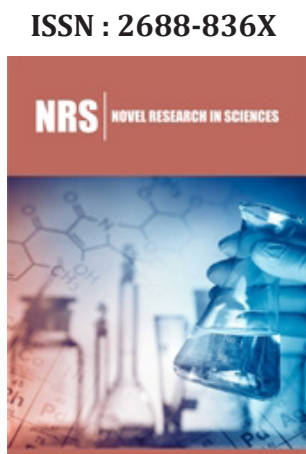

*Corresponding author: Klemen Bedenčič, Orthopaedic Department General Hospital, Novo Mesto Šmihelska cesta 1 8000 Novo Mesto, Slovenia

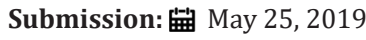

Published: 漹 June 10, 2019

Volume 1 - Issue 1

How to cite this article: Klemen Bedenčič, Matevž B. Chronic Achilles Tendon Rupture Reconstruction with a Endoscopic Flexor Hallucis Longus Transfer: A Case Re-Port. Nov Res Sci.1(1). NRS.000502.2019.

DOI: 10.31031/NRS.2019.1.000502

Copyright@ Klemen Bedenčič, This article is distributed under the terms of the Creative Commons Attribution 4.0 International License, which permits unrestricted use and redistribution provided that the original author and source are credited.

\author{
Klemen Bedenčič** and Matevž Bajuk \\ Orthopaedic Department General Hospital, Slovenia
}

\begin{abstract}
Chronic Achilles tendon (AT) rupture is often undiagnosed disease especially in elderly. Management of chronic AT ruptures is different from acute ruptures as tendon ends are retracted and atrophied with lots of fibrous ends. The results and outcome of chronic rupture is fairly unsatisfactory. We present our first case report of treating chronic AT rupture with large defect and fatty infiltrated muscle tissue using a posterior ankle endoscopy for flexor hallucis longus (FHL) transfer.
\end{abstract}

\section{Case Report}

We present a case with 86 year old woman with difficulties of walking and debilitating pain in right foot and weakness during plantar flexion in the right foot for over 2 years. She was unaware of traumatic incidence. Physical examination showed an Achilles ten-don gap under the skin at inspection and upon palpation of the area. She was unable to raise her heel off the ground and Thompson test was positive. An US image and MRI was assessed for detailed diagnosis. A chronic full-thickness rupture of AT was seen on both imaging tools with $15,5 \mathrm{~cm}$ large defect and fat infiltration of split ends.

We indicated operation with FHL transfer using endoscopy rather than open augmented procedure as we would avoid large skin incision and a possibility of wound healing problems. We performed an operation in prone position under general anesthesia with a thigh tourniquet. Standard posterior ankle endoscopy was performed for identifying and clearing the FHL tendon. A medial foot incision for a final harvest of FHL tendon was made. The FHL tendon is released as it enters in its tunnel beneath the sustentaculum tali; a tunnel is then drilled in the calcaneus as near to the AT insertion as possible. By use of a suture-passing device, the free end of the FHL is advanced to the plantar aspect of the foot. After adequate tension is applied to the construct, the tendon is fixed in place with an interference screw in an inside-out fashion. This minimally invasive approach is a safe and valid alternative to classic open procedures with the obvious advantages of pre-serving the soft-tissue envelope and using a biologically intact tendon [1].

A non-weight-bearing short leg cast in the equinus position was then used for 2 weeks, followed by a new short leg cast in a less equinus position to recover the plantigrade position for three weeks. After the cast was removed, a physical therapy program was prescribed for 3 weeks to recover the correct plantigrade position as well as tip-toed walking. She was able to return to normal activities within a month after cast removal.

\section{Discussion}

The Achilles tendon is the strongest and largest tendon in the human body per tensile strength. Despite its functional role to withstand large forces, it is one of the tendons most commonly affected by spontaneous rupture [2]. Even though Achilles tendon ruptures are frequent and are usually not difficult to diagnose for experienced physicians, more than $20 \%$ of acute injuries are misdiagnosed, leading to a chronic rupture. A chronic rup-ture can be defined as a rupture with a delay in diagnosis or treatment for more than 6 weeks [3].

The treatment of chronic Achilles tendon rupture is a challenge for most orthopaedic surgeons [4]. It is different from the acute Achilles tendon rupture. Chronic Achilles tendon 
ruptures with large gaps may lead to ankle dysfunction, gait disturbances and limping [5].

Several different techniques have been described for the treatment of chronic or neglected Achilles tendon ruptures in adults, such as bridging approximation procedures, V-Y slide lengthening of the tendon, gastrocnemius advancement, fascial turndown flaps, free tissue transfer, and the use of synthetic grafts $[6,7]$.

A chronically degenerated reconstructed AT tendon is expected to re-rupture with a compromised biological value. Thus the use of a biologically intact tendon of through its transfer is an appealing option.

Flexor hallucis longus (FHL) transfer is a well-established treatment option in failed Achilles tendon (AT) repair and has been routinely performed as an open procedure $[7,8]$.

As for the FHL harvest, transfer morbidity is clinically insignificant, even for good push-off or balance in young active adults [9]. Our minimally invasive technique for FHL transfer further eliminates morbidity from this procedure, such as wound healing-related problems. It is a safe and valid alternative to classic open augmentation procedures with a good expected clinical outcome $[10,11]$.

Routine FHL transfer as first option should not be recommended for younger patients as it can compromise a function of halluces, even though FHL has good adaptation capacity and it can hypertrophy up to $50 \%[12,13]$.

In our case we have achieved an active range of motion with good plantar function for walking and stair climbing. A physical therapy program was needed after cast removal to recover the full range of ankle motion. By 3 months after the surgery an excellent functional status was noticed. The incision had healed completely and the foot was plantigrade, the patient could walk on tip toes, and she was able to perform 20 repetitive single-leg heel-raise manoeuvres. At the latest follow-up 6 months after surgery, the patient had recovered sufficient range of motion of the ankle and ankle function for normal plantar flexion.

\section{Conclusion}

Various repair techniques have been reported for chronic Achilles tendon rupture. Optimal treatment of chronic Achilles tendinopathy, with or without rupture, remains challenging [14]. The diagnosis is more difficult, and management is technically more demanding than primary repair of acute rupture [15]. Complications, such as wound breakdown and infection, are also frequent following open procedures. Minimally invasive reconstruction of chronic Achilles tendon ruptures provides good clinical results, with the advantages of decreased perioperative morbidity and complications rate. Reconstruction with the tendon of flexor hallucis longus using endoscopy is safe, less invasive, and reliable, performed in patients with low-to-moderate demands as we regain $80-85 \%$ of Achilles tendon power. In our case and endoscopic FHL reconstruction was a better choice as an open augmented procedure, since patient was elderly and wound healing would be a problem.

\section{Ethical Standards}

The patient approved the publication of this case report.

\section{References}

1. Maffulli N, Ajis A (2008) Management of chronic ruptures of the Achilles tendon. J Bone Joint Surg Am 90:1348-1360.

2. Movin T, Ryberg Å, McBride DJ, Maffulli N (2009) Acute rupture of the achilles tendon. Foot and Ankle Clinics 10(2): 331-356.

3. Maffulli N (1999) Rupture of the Achilles tendon. J Bone Joint Surg Am 81(7): 1019-1036.

4. Cetti R, Christensen SE, Ejsted R, Jensen NM, Jorgensen U, et al. (1993) Operative versus nonoperative treatment of Achilles tendon rupture. A prospective randomized study and review of the literature. American Journal of Sports Medicine 21(6): 791-799.

5. Clelland D, Maffulli N (2004) Neglected rupture of the Achilles tendon: reconstruction with peroneus brevis tendon transfer. Surgeon 2(4): 209-213.

6. Padanilam TG (2009) Chronic Achilles tendon ruptures. Foot Ankle Clin 14: 711-728.

7. Flint JH, Wade AM, Giuliani J, Rue JP (2014) Defining the terms acute and chronic in orthopaedic sports injuries: a systematic review. American Journal of Sports Medicine 42(1): 235-241.

8. Maffulli N, Via AG, Oliva F (2015) Chronic Achilles tendon disorders: tendinopathy and chronic rupture. Clinics in Sports Medicine 34(4): 607-624.

9. Coull R, Flavin R, Stephens MM (2003) Flexor hallucis longus tendon transfer: Evaluation of postoperative morbidity. Foot Ankle Int 24: 931934.

10. Hahn F, Meyer P, Maiwald C, Zanetti M, Vienne P (2008) Treatment of chronic Achilles tendinopathy and ruptures with flexor hallucis tendon transfer: Clinical outcome and MRI findings. Foot Ankle Int 29: 794-802.

11. Gonçalves S, Caetano R, Corte-Real N (2015) Salvage Flexor Hallucis Longus Transfer for a Failed Achilles Repair: Endoscopic Technique. Arthrosc Tech 4(5): e411-e416.

12. Oksanen MM, Haapasalo HH, Elo PP, Laine HJ (2014) Hypertrophy of the flexor hallucis longus muscle after tendon transfer in patients with chronic Achilles tendon rupture. Foot and Ankle Surgery. 20(4): 253257.

13. Coull R, Flavin R, Stephens MM (2003) Flexor hallucis longus tendon transfer: evaluation of postoperative morbidity. Foot and Ankle International 24(12): 931-934.

14. Leslie HD, Edwards WH (2005) Neglected ruptures of the Achilles tendon. Foot Ankle Clin 10(2): 357-370.

15. Flint JH, Wade AM, Giuliani J, Rue JP (2014) Defining the terms acute and chronic in orthopaedic sports injuries: a systematic review. American Journal of Sports Medicine 42(1): 235-241.

For possible submissions Click below: 